\title{
The perceptual basis of loudness ratio judgments
}

\author{
BRUCE SCHNEIDER \\ University of Toronto, Toronto, Ontario, Canada \\ SCOTT PARKER \\ The American University, Washington, D.C. 20016 \\ GLENN FARRELL \\ Syracuse University, Syracuse, New York 13210
}

and

GARY KANOW

University of Pennsylvania, Philadelphia, Pennsylvania 19104

\begin{abstract}
In. Experiment 1, subjects were required to estimate loudness ratios for 45 pairs of tones. Ten $1,200-\mathrm{Hz}$ tones, differing only in intensity, were used to generate the 45 distinct tone pairs. In Experiment 2 , subjects were required to directly compare two pairs of tones (chosen from among the set of 45) and indicate which pair of tones had the greater loudness ratio. In both Experiments 1 and 2, the subjects' judgments were used to rank order the tone pairs with respect to their judged loudness ratios. Nonmetric analyses of these rank orders indicated that both magnitude estimates of loudness ratios and direct comparisons of loudness ratios were based on loudness intervals or differences where loudness was a power function of sound pressure. These experiments, along with those on loudness difference judgments (Parker \&Schneider, 1974; Schneider, Parker, \& Stein, 1974), support Torgerson's (1961) conjecture that there is but one comparative perceptual relationship for loudnesses, and that differences in numerical estimates for loudness ratios as opposed to loudness intervals simply reflect different reporting strategies generated by the two sets of instructions.
\end{abstract}

A continuing difficulty for proponents of a power law representation for the growth of loudness is the nonlinear relationship between judgments based on loudness differences and judgments based on loudness ratios. For example, in a magnitude estimation task, observers are asked to assign numbers to stimuli such that the ratio of any two numbers is the ratio of the loudnesses of the corresponding sounds. Numerical judgments obtained in this fashion are found to be a power function of sound pressure, i.e.,

$$
\mathrm{N}=\mathrm{kI}^{0.6}
$$

where $\mathrm{N}$ represents the observer's numerical judgment, I denotes sound pressure, and $\mathrm{k}$ is a constant of proportionality. Experimental evidence collected by Stevens and others (see S. S. Stevens, 1971; Marks, 1974, for recent reviews) has, in general, supported the power law representation for the growth of loudness, although it has long been known that a second class of experiments, involving judgments of loudness differences, do not yield the same representation for loudness. For example,

This research was supported in part by NSF Grant GB 36211 . and in part by a grant from the National Research Council of Canada. Reprints may be obtained from Bruce Schneider. Department of Psychology. Erindale College. University of Toronto. when observers are required to assign numbers to sounds in a category scaling task, such that the numbers represent the loudness differences among the sounds rather than loudness ratios, the loudness scale values obtained in this fashion are a negatively accelerated function of the loudness scale values obtained via ratio estimation techniques (Eisler, 1962; Schneider \& Lane, 1963; S. S. Stevens, 1960; and S. S. Stevens \& Guirao, 1962). This is counter to the linear relationship we would expect if subjects could judge both loudness differences and loudness ratios accurately. 1

S. S. Stevens (1971) accounts for this nonlinear relationship by arguing that judgments of sensory differences yield a biased or distorted representation of the loudness continuum. He claims that subjects are unable to judge accurately equal loudness intervals along the entire range of the loudness continuum. According to Stevens, a constant loudness difference seems larger near the lower end than near the higher end of the continuum. This kind of asymmetrical distortion, if it truly existed, would explain the negative acceleration of the category scale when plotted against the magnitude scale.

A different account of the relationship between the two kinds of judgments is provided by Torgerson (1961). Torgerson noted that, in magnitude estimation, subjects are asked to judge loudness ratios for pairs of 
stimuli, while in category estimation they are asked to give estimates of the loudness differences between sounds. To do this successfully, Torgerson pointed out, subjects have to be able to judge two distinct relation. ships along a sensory continuum; that is, they must be able to judge the loudness difference between two sounds as well as the loudness ratio of one sound to another. Torgerson's conjecture was that they could not do both. He argued that there was only one perceptual relationship defined on a pair of stimuli from an intensive continuum. Thus, the numbers that a subject would assign for pairs of stimuli equally separated with respect to this subjective relationship would depend on the instructions. In a magnitude estimation task, the numbers assigned to these equally separated pairs would have equal numerical ratios between them, while in a category experiment, the numbers assigned to these equally separated pairs would have equal numerical differences. The result would be a logarithmic relationship between category and magnitude scales of loudness.

Unfortunately, for Torgerson's account, a logarithmic relationship between category and magnitude scales of loudness does not quite describe the data. While the function describing the data is negatively accelerated, it deviates systematically from a logarithmic form (Schneider \& Lane, 1963; S. S. Stevens, 1960). Furthermore, S. S. Stevens and Galanter (1957) have shown that the degree to which a logarithmic relationship does hold depends on a number of experimental parameters.

On the other hand, it is extremely difficult to test, in any rigorous fashion, whether or not Stevens' account of the reasons why the two scales are nonlinearly related is truly descriptive of the kinds of bias which occur in loudness difference judgments. As a result, Marks (1974), in considering a large number of studies concerning the two kinds of judgments, came to the conclusion that it was better to speak of two loudness scales: one for difference judgments and the procedures upon which they are based, and one for ratio judgments and the procedures on which they are based. However, many investigators are understandably reluctant to give up the notion that there is only one representation for loudness and that judgments of sensory differences and sensory differences and sensory ratios reflect different aspects of the one underlying loudness scale.

It is possible that some of the difficulties involved in resolving this issue result from the experimenter's reliance on numerical judgments on the part of observers. In order for ratio and difference judgments to yield the same loudness scale, it must be assumed that, in ratio estimation tasks, judgments of loudness ratios are log interval scale measurements of loudness ratios, while, in the interval scaling tasks, judgments of loudness differences are ratio scale measurements of loudness differences. If, for any reasons, observers' judgments were biased or distorted in some fashion, then one or both of these assumptions would no longer be true, and it would be extremely difficult to determine the true relationship between judgments of loudness differences and judgments of loudness ratios. Hence, to be able to determine the actual relationship between judgments of loudness ratios and loudness differences, nonlinearities in the subject's numerical estimates must be eliminated or taken into account when loudness scale values are assigned to the tones being judged. In the present experiments, nonmetric scaling techniques are employed to analyze both loudness difference and loudness ratio judgments. The advantage in the nonmetric techniques is that they make less stringent assumptions about the relationship between the subject's judgments and actual ratios and differences along the sensory continuum. Hence, monotonic biases in the subjects' reporting strategy will not affect the determination of the relationship between judgments of loudness ratios and judgments of loudness differences.

Experiments which have used nonmetric techniques to analyze judgments of loudness differences have been performed by Parker and Schneider (1974) and Schneider, Parker, and Stein (1974). In one experiment, Parker and Schneider had subjects give magnitude estimates of loudness differences for tonal pairs. The 10 tones used to construct the 45 tone pairs used in that experiment were identical in frequency and ranged in intensity from 50 to $104 \mathrm{~dB}$. For purposes of data analysis, the loudness values of the tones were conceived of as points on a line segment since loudness is a unidimensional experience. Magnitude estimates of loudness difference then were regarded as analogous to estimates of distance along this line segment. Shepard (1966) has shown that, provided the number of stimuli is $\geqslant 10$, the rank order of interpoint distances can be used to determine projection values along the line segment which are, for all practical purposes, unique up to addition and multiplication of a constant. The rank order of loudness difference judgments was used to determine projections along a line segment for the 10 tones. These projection values presumably represented an interval scale of loudness. Parker and Schneider showed that, when these interval scale values were suitably adjusted by an additive constant, loudness was a power function of sound pressure with an exponent of 0.26 . Note that in order to obtain this interval scale of loudness, it was only necessary to assume that loudness difference judgments were monotonically related to the actual loudness differences atong the loudness continuum. Thus. the stronger assumption. that loudness difference judgments were directly proportional to loudness differences was avoided.

Schneider et al. (1974) also found that a 0.26 power function representation of loudness was necessary in order to account for direct comparisons of sensory intervals. Subjects in this experiment were required to directly compare pairs of tones and indicate which 
pair of tones had the greater loudness difference. This, of course, requires only a binary decision on the part of the subject, i.e., either the tones in pair A or pair B are judged to be more widely separated in loudness. From these binary comparisons, a rank order of loudness differences for the tones was constructed. A nonmetric analysis of this rank order indicated that loudness grew as the 0.26 power of sound pressure. Note that this analysis assumes only that a subject can accurately report which of two loudness intervals is the greater.

These nonmetric scaling techniques can be used to study judgments of loudness ratios as well as loudness difterences. In the tirst experiment reported here, magnitude estimates of the loudness ratios for the 45 pairs of tones employed in the Parker and Schneider (1974) study were obtained from subjects. In a second experiment. subjects directly compared pairs of tones and indicated which pair of tones had the larger loudness ratio. If Torgerson is correct, and subjects can judge but one comparative relationship along a sensory continuum, then the rank order of loudness ratio judgments obtained in these two experiments should be identical to that obtained in the Parker and Schneider (1974) and the Schneider et al. (1974) studies. If, on the other hand, subjects could judge both differences and ratios of loudnesses, then the rank order of tone pairs obtained from magnitude estimates and direct comparisons of loudness ratios should be systematically different from that obtained when subjects judge loudness differences. Furthermore, if these orderings of loudness differences and loudness ratios are monotonically related to the actual loudness differences and loudness ratios along the loudness continuum, then it is possible to construct a ratio scale of loudness from these rank orders (Krantz, Luce, Suppes, \& Tversky, 1971). The present experiments, then, will enable us to determine whether Torgerson's conjecture, or the S. S. Stevens (1971) or Marks (1974) account of the relationship between judgments of loudness differences and loudness ratios, is correct.

\section{METHOD}

\section{Experiment 1}

Subjects. Four graduate students in the Department of Psychology and one in the Department of English and Comparative Literature, Columbia University, served as subjects. Their ages ranged from 22 to 27 years. All subjects claimed to have normal hearing. Three had previous experience in magnitude estimation experiments, but none had ever estimated loudness differences.

Apparatus. Calibrations and listening conditions were identical to those used by Carvellas and Schneider (1972), except that the subjects sat in an Industrial Acoustics sound-resistant booth, Model 300.

Procedure. Each subject served in four experimental sessions. The stimuli in the first three sessions were the $\mathbf{4 5}$ pairs of unequal tones constructible from the $101,200-\mathrm{Hz}$ tones with intensities of 50,56 , 60.68, 72, 80, 86, 94, 98, and $104 \mathrm{~dB}$ re .0002 dynes $/ \mathrm{cm}^{2}$. The stimuli in the fourth session were those 10 tones played singly.
Just prior to the first session, the subjects were instructed as follows: "This is an experiment on your perception of loudness. You will hear pairs of tones. The tones in a pair will differ in loudness. Your task is to determine the ratio of the loudnesses of the tones in each pair. So. for example, if the first tone in a pair sounds ten times as loud as the second, you should say 'ten.' If the first tone in some other pair sounds half as loud as the second, you should say 'one-half.' You may use any positive number-integer, fraction, or decimal. You may not use negative numbers or zero. Are there any questions?"

The subject was then led in to the booth and shown how to operate the switch that presented the tones. and how to wear the earphones. During the first session. the $\mathbf{4 5}$ tone pairs were played in jrregular order. The subject listened to each pair as long as he wished and then spoke a number. In each of Sessions 2 and 3, the 45 tone pairs were presented in two irregular sequences separated by a 5-min break. The sequences in the second and third sessions were such that each tone pair appeared twice before and twice after each other tone pair. Also, each tone appeared equally often on each operative position of the switch. The second sequence of the third session was identical to that used in the first session.

In the fourth session, subjects were required to magnitude estimate the loudnesses of each of the 10 tones. The instructions for this session were similar to those of S. S. Stevens (1956). Each of the 10 tones was presented 5 times each in an irregular order.

Listening was monaural (right ear) throughout all four sessions.

\section{Experiment 2}

Subjects. All eight subjects were graduate students at Columbia University. All had had some experience with magnitude- or ratio-estimation experiments. Three had served in Experiment $I$. None had ever been asked to estimate loudness differences.

Apparatus. A full description of the tone generation and control equipment may be found in Schneider et al. (1974). The subject presented himself with one of two pairs of tones to be compared by pressing Button 1. Button 2 resulted in the presentation of the other tone pair. The tones in a pair were $750 \mathrm{msec}$ in duration and were separated by a "dead" period of $900 \mathrm{msec}$. The subject was allowed to present himself with pairs as many times as he wished in order to arrive at a decision. Signal frequency and voltage were monitored with a Dymec Electronic counter, Model Dy-2501, and a calibrated Ballantine VTVM. Oscillographic examination of signal waveform for each test tone revealed no apparent distortion or clipping. Voltage levels were converted to sound pressure via earphone calibrations performed by the National Bureau of Standards in Washington, D.C., with an NBS 9-A coupler. Listening was monaural (right ear) throughout the entire experiment. The experimenter and subject communicated via an intercom.

Procedure. The subject, who was seated in an IAC sound-resistant booth (Model 300) was instructed to report which of two pairs of tones contained the larger loudness ratio. The 45 tone pairs to be compared were the same as in Experiment 1. If every pair of tones were compared to every other pair. then 990 pair comparisons would have been necessary. Schneider et al. (1974), however, have shown that only 540 of these 990 comparisons are necessary in order to rank order the 45 tone pairs along the loudness ratio dimension. Furthermore, they showed that it was possible, given these 540 comparisons, to determine the extent to which these judgments satisfy the two critical axioms of a positive difference structure (Krantz et al.. 1971). These two axioms must be satisfied if interval scale measurement of loudness ratios is to be achieved.

During the course of eight sessions, the subject was presented once with each of 540 pairs of tones. Prior to the eight test sessions. each subject served in a practice session containing 70 tone-pair comparisons. The first seven test sessions contained 70 tone-pair comparisons, and the eighth, of course, contained 50. Sessions generally lasted 45-70 min, with a 10-min break halfway through the session.

The 540 pairs of tone pairs were presented in a different random order for each of the eight subjects. The four tones presented in each 
comparison of pairs occurred in eight different arrangements. Within a pair, there were two orders which could occur: (1) $a, b$ or (2) b,a. Since two pairs were involved, this meant that there were four possible orderings: (1) $a, b: x, y$; (2) $b, a: x, y$; (3) $a, b: y, x$; or (4) b,a:y,x. Since a pair of tones could occur on either Button 1 or Button 2, this allowed for eight arrangements. These eight arrangements for a comparison of tone pairs were assigned randomly across the eight subjects to counterbalance for order effects.

\section{RESULTS}

\section{Experiment 1}

In the first experiment, subjects judged the loudness ratios of the 45 tone pairs. Prior to averaging, reciprocals were taken for the tone pairs in which the more intense tone appeared second, since these tone pairs received ratio judgments of less than 1. The first of the five judgments was discarded and the geometric mean of the remaining four estimates was computed for each of the 45 tone pairs. The geometric mean was chosen as a measure of central tendency, since the variance of the ratio estimates generally increased with the mean. The geometric means were then ranked, within subjects, from 1 to 45. Kendall's coefficient of concordance, W (Siegel, 1956, pp. 229-238), for these five rank orders was found to be 0.91 . Thus, agreement among subjects as to the rank order of loudness ratio judgments was good.

The arithmetic mean of the ranks across the five subjects was computed for each stimulus pair to obtain the average rank of the loudness ratio judgments over the 45 tone pairs. Taking average rank in this fashion is equivalent to assuming that judgments of loudness ratios $\left(\mathbf{R}_{\mathrm{ij}}\right)$ are a monotonic function of the ratios of the loudnesses of the corresponding tones in a pair, i.e., $R_{i j}=m\left(L_{i} / L_{j}\right)$, where $L_{i}$ is the psychological loudness of tone $i$ and $m$ represents a monotonic relationship between the two variables. It is clear that if the ratio judgments are a monotonic function of loudness ratios, they are also a monotonic function of the logarithm of the loudness ratios, and therefore a monotonic function of the logarithmic difference between the loudnesses of the two tones; that is,

$$
\begin{aligned}
\mathrm{R}_{\mathrm{ij}}=\mathrm{m}\left(\mathrm{L}_{\mathrm{i}} / \mathrm{L}_{\mathbf{j}}\right) & =\mathrm{m}\left[\log \left(\mathrm{L}_{\mathbf{i}} / \mathrm{L}_{\mathbf{j}}\right)\right] \\
& =\mathrm{m}\left(\log \mathrm{L}_{\mathbf{i}}-\log \mathrm{L}_{\mathbf{j}}\right)
\end{aligned}
$$

Hence, if loudness ratio judgments are a monotonic function of the ratio of the loudnesses of the individual tones, the rank order of the loudness ratio judgments is the same as the rank order of the difference between the logarithms of the loudnesses of the tones in a pair. This rank order was analyzed nonmetrically to determine a set of projection values along a line segment whose differences would best predict this rank order. Since $1,200-\mathrm{Hz}$ tones vary minimally in pitch over the range of intensities used
(S. S. Stevens, 1935), the basis for all judgments was presumed to be loudness variation among the tones. Hence, the analysis was unidimensional, and the tone intensities in decibels were used as the starting configuration in the nonmetric computer program (Carvellas \&Schneider, 1972).

For purposes of this analysis, loudness ratio judgments were considered as estimates of the differences in log loudness for the tones in a pair. Hence, if subjects were truly judging loudness ratios, the projection value of a tone in this experiment represented the log loudness of the tone, and the difference between any two projection values represented the predicted logarithmic difference in loudness between the two tones. Consequently, the nonmetric program attempts to find projection values for the tones such that differences in projection values for tone pairs are monotonically related to the judged loudness ratios.

Stress, Kruskal's (1964) measure of goodness of fit, was computed for the output of the nonmetric program and was found to be $3.1 \%$. Stress measures the discordance between the predicted distances, ds, and a set of distances, ds, that are (a) monotonically related to the rank order of loudness ratio judgments, and (b) as much like the ds as they can be within the restrictions imposed by (a). Stress is given by $\left[\Sigma(\mathrm{d}-\hat{\mathrm{d}})^{2} / \mathrm{d}^{2}\right]^{1 / 2}$, often expressed as a percentage. Notice that perfect ordinal agreement produced $\mathrm{d}=$ $\hat{\mathrm{d}}$, and in this case, stress $=0$. Kruskal (1964) states that stress values of $5 \%$ or less indicate "good" agreement between the ds and ds.

The index of metric determinacy (M), which was originally developed by Shepard, was estimated from Young's (1970) nomogram. $M$ is the squared Péarson correlation coefficient between the true distances (whose rank ordering serves as the input to the algorithm) and the ds produced by the algorithm. Hence, $M$ varies between 0 and 1 , and $M=1$ means that the true distances have been perfectly reconstructed. In no empirical investigation using these techniques are the true distances known, but Young provides a nomogram for estimating $M$ from the number of points, number of dimensions, and stress-all of which are available. The result is that, in nonmetric scaling analyses, if $M$ is sufficiently high (above 0.98 , say) the point coordinates produced by the algorithm are properly regarded as an interval scale representation of the original points. In this experiment, $M$ was, conservatively, 0.99. Thus the projection values achieved from the nonmetric program constitute an interval scale representation.

If subjects' judgments of loudness ratios are a monotonic function of the loudness ratio of one tone to another, then the rank order of ratio judgments is a rank order of the differences between the logarithms of loudnesses of the two tones. This, in turn, implies that the projection values from the nonmetric analysis 
represent an interval scale of $\log$ loudness, i.e., $\log \mathrm{L}$ $=a P+b$, where $L$ is loudness, $P$ are the projection values from the nonmetric analysis of the ratio judgments. and $\mathbf{a}$ and $b$ are constants. If loudness is $a$ power function of sound pressure, then $\log i=$ $\log \left(\mathrm{kI}^{\mathrm{n}}\right)=\mathrm{n} \log (\mathrm{I})+\mathrm{k}^{\prime}=\mathrm{aP}+\mathrm{b}$, where $\mathrm{I}$ represents sound pressure and $\mathrm{k}^{\prime}=\log \mathrm{k}$. An equivalent expression is $P+b / a=(n / a)$ $\log I+k^{\prime} / a$ or $P=(n / a) \log I+c$, where $c=$ $\mathrm{k}^{\prime} / \mathrm{a}-\mathrm{b} / \mathrm{a}$. Hence, if judgments of loudness ratios are a monotonic function of loudness ratios and if a power function relates loudness to sound pressure, then the projection values obtained for ratio judgments from the nonmetric program should be a logarithmic function of sound pressure.

Figure 1 plots the projection values obtained from the nonmetric program as a function of decibels of sound pressure. Since decibels are a $\log$ function of sound pressure. a straight line in these coordinates would be expected if loudness ratio judgments were monotonic with loudness ratios and a power function described the relationship between loudness and sound pressure. Note that the points deviate significantly from the best fitting straight line (method of least squares) in these coordinates. These data suggest, then, that either (1) subjects' judgments of loudness ratios are not monotonic with loudness ratios. or (2) a power function does not describe the growth of loudness.

Suppose. however. that Torgerson was correct and that subjects could judge only one perceptual relationship along a prothetic continuum, and that the relationship was a loudness difference relationship. Then judgments of loudness ratios

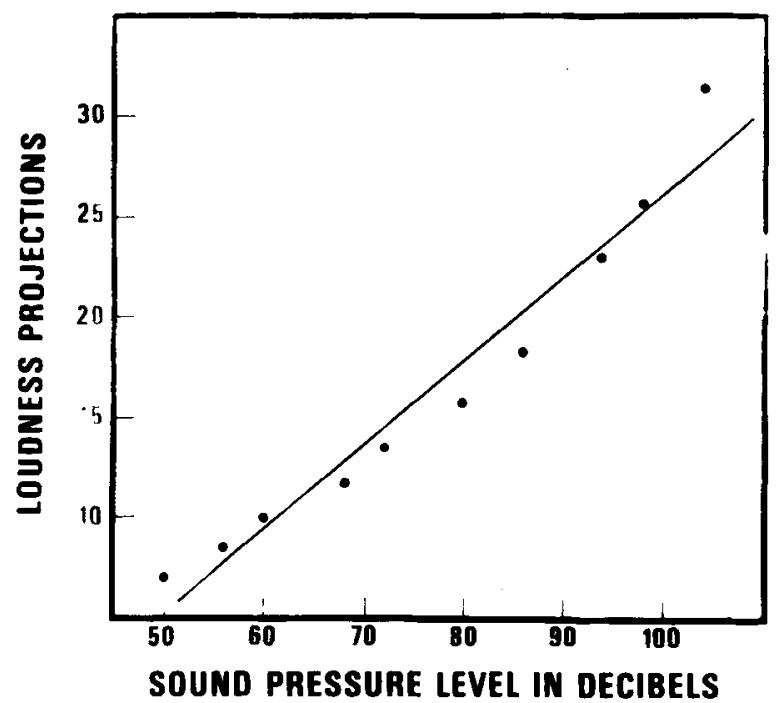

Figure 1. Loudness projections for 10 tones as a function of sound pressure level in decibels. The loudness projections were obtained from a nonmetric analysis of estimates of loudness ratios. A straight line in these coordinates would indicate that loudness ratio judgments were based on loudness ratios where loudness is a power function of intensity.

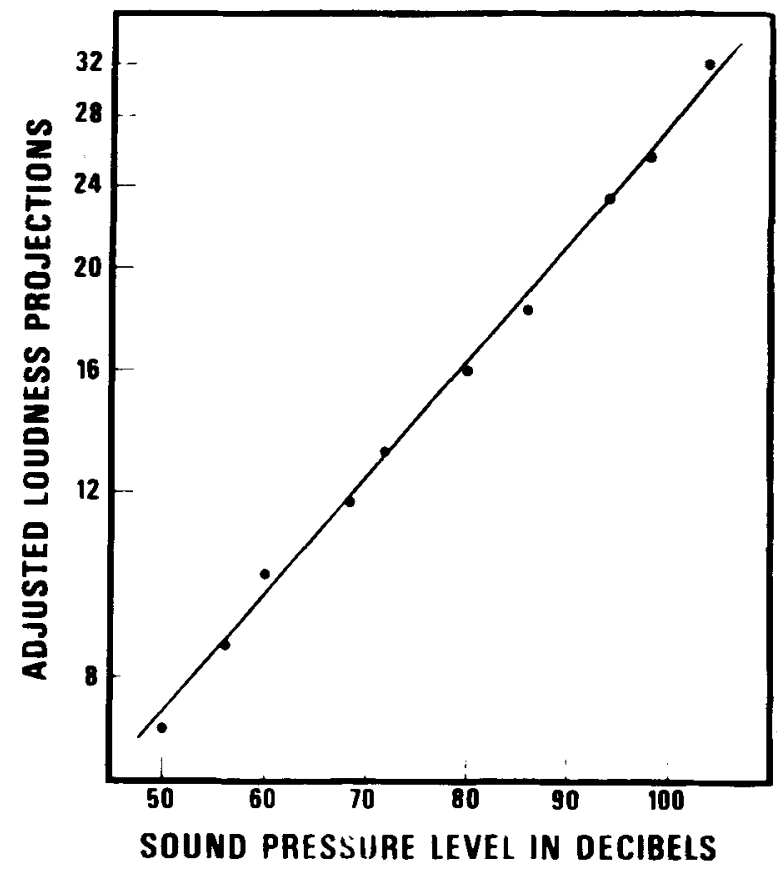

Figure 2. Adjusted loudness projections for 10 tones as a function of decibels of sound pressure. Notice that the ordinate is spaced logarithmically. The adjusted loudness projections were obtained from a nonmetric analysis which assumed that judgments of loudness ratios were based on loudness differences. A straight line in these coordinates indicates that loudness ratios were based on loudness differences where loudness is a power function of intensity.

should. at the very least. be monotonically related to judgments of loudness differences. If judgments of loudness ratios were monotonic with loudness differences, then we would have $R_{i j}=m\left(D_{i j}\right)=$ $m\left(L_{i}-L_{j}\right)$, where $D_{i j}$ represents a judgment of the loudness difference between tones $i$ and $j$. In a nonmetric analysis of loudness difference judgments, it is assumed that the rank order of estimates of loudness difference is analogous to a rank of distances along a line segment. Hence. these projection values would represent an interval scale of loudness. i.e., L $=\mathrm{aP}+\mathrm{b}$. If loudness is a power function of intensity, then $a P+b=L=k I^{n}$. Equivalently, $P+b^{\prime}=k^{\prime} l^{n}$, where $b^{\prime}=b / a$ and $k^{\prime}=k / a$. Taking $\log$ of both sides yields $\log \left(\mathrm{P}+\mathrm{b}^{\prime}\right)=$ $\mathrm{n} \log \mathrm{l}+\mathrm{c}$. where $\mathrm{c}=\log \mathrm{k}^{\prime}$. Thus, if there is $\mathbf{a} \mathbf{b}^{\prime}$ such that $\log \left(P+b^{\prime}\right)$ is a linear function of decibels of sound pressure, the present data are consistent with the notion that subjects, when asked for loudness ratios, actually report on loudness differences, where loudness is a power function of sound pressure. Therefore, a value of $b^{\prime}$ was determined that maximized the squared Pearson correlation coefficient $\left(\mathbf{r}^{2}\right)$ between $\log \left(\mathbf{P}+\mathbf{b}^{\prime}\right)$ and decibels. The projection values adjusted by the value of $b^{\prime}$ which maximized $r^{2}$ are referred to as the adjusted loudness projections.

Figure 2 plots the $\log$ of the adjusted loudness projections against decibels of sound pressure. A 
Table 1

Squared Correlation Coefficients and Exponents for the Five Subjects Magnitude Estimating the Loudnesses of the Individual Tones

\begin{tabular}{ccc}
\hline Subjects & $\mathrm{I}^{2}$ & Exponent \\
\hline S.K. & .986 & .56 \\
L.K. & .947 & .68 \\
B.R. & .954 & .68 \\
M.T. & .970 & .68 \\
R.H. & .954 & .37 \\
\hline
\end{tabular}

straight line (method of least squares) is drawn through the points. The linearity of the points in these coordinates suggests that loudness ratio judgments were actually based on loudness differences where loudness was a power function of sound pressure. The estimate of the exponent of the power function for these data (obtained from the slope of the line in Figure 2) is 0.23 . These data suggest that, when the subjects in this experiment were asked to judge loudness ratios, those judgments were monotonically related to differences between the 0.23 power of the sound pressures of the individual tones; i.e., $R_{i j}=$ $\mathrm{m}\left(\mathrm{I}_{\mathrm{i}}^{0.23}-\mathrm{I}_{\mathrm{j}}^{0.23}\right)$.

To check whether there was anything unusual about this particular group of subjects, they were asked to magnitude-estimate the loudnesses of the 10 tones used in the loudness ratio experiment above. The first of five estimates was discarded, and the geometric mean of the remaining four estimates was computed. These mean estimates were plotted against stimulus intensity in decibels, and $r^{2}$ was computed for each subject. Values of $r^{2}$ and of the exponent of the best fitting power function relating mean estimates and sound pressures are shown in Table 1. These data are typical of those commonly found for individual subjects (J. C. Stevens \& Guirao, 1964). In Figure 3, the geometric mean estimate for each intensity (averaged across the five subjects) is plotted as a function of sound pressure level in $\log$-log coordinates. The best fitting straight line (method of least squares) has a slope (exponent of the power function) of 0.60 and provides a good fit to the data $\left(r^{2}\right.$ $=0.989$ ). This value of the exponent is quite close to that of 0.54 reported by Hellman and Zwislocki (1963) for the growth of monaural loudness vs. sound pressure for $1,000-\mathrm{Hz}$ tones. Thus, the subjects in the present experiment exhibit quite ordinary performance when judging the loudnesses of individual tones using the technique of magnitude estimation.

\section{Experiment 2}

In this experiment, eight observers judged which of two pairs of tones had the greater loudness ratio for each of the 540 comparisons conducted in this study. For each of the 540 tone-pair comparisons, the number of people who judged pair $A$ to have a larger loudness ratio than pair $B$ was recorded. Majority rule determined which pair was to have the higher ranking with respect to the loudness ratios of the pairs. i.e., if tive or more subjects chose pair $B$ as having a larger loudness ratio than pair $A$, then $B$ should have a higher location than pair $A$ in the final rank ordering of the $\mathbf{4 5}$ pairs. If precisely four of the eight subjects chose pair $A$ and four chose pair $B$, then no constraint was placed by this particular comparison on their position vis à vis one another in the final rank order. There were 32 such ties in the 540 comparisons. of course, the constraints placed by the other comparisons were sufficient to determine their order in the ranking of pairs with respect to loudness ratios.

It was not possible to construct a single ranking of the $\mathbf{4 5}$ pairs such that a higher rank was assigned to pair $A$ than to pair $B$ whenever pair $A$ was judged by five or more observers to have a greater loudness ratio than pair B. The reason for this is that there were occasional violations of weak transitivity, i.e., there were cases in which pair $A$ was judged to have a larger ratio than pair $B$, pair $B$ was judged to have a larger ratio than pair $C$, but pair $C$ was judged to have a larger ratio than pair $A$. In instances such as these, any of the three possible orderings $[(A, B, C),(B, C, A)$, $(C, A, B)]$ is inconsistent with one of the binary comparisons of pairs obtained from the subjects. The existence of an intransitive triplet guarantees that no

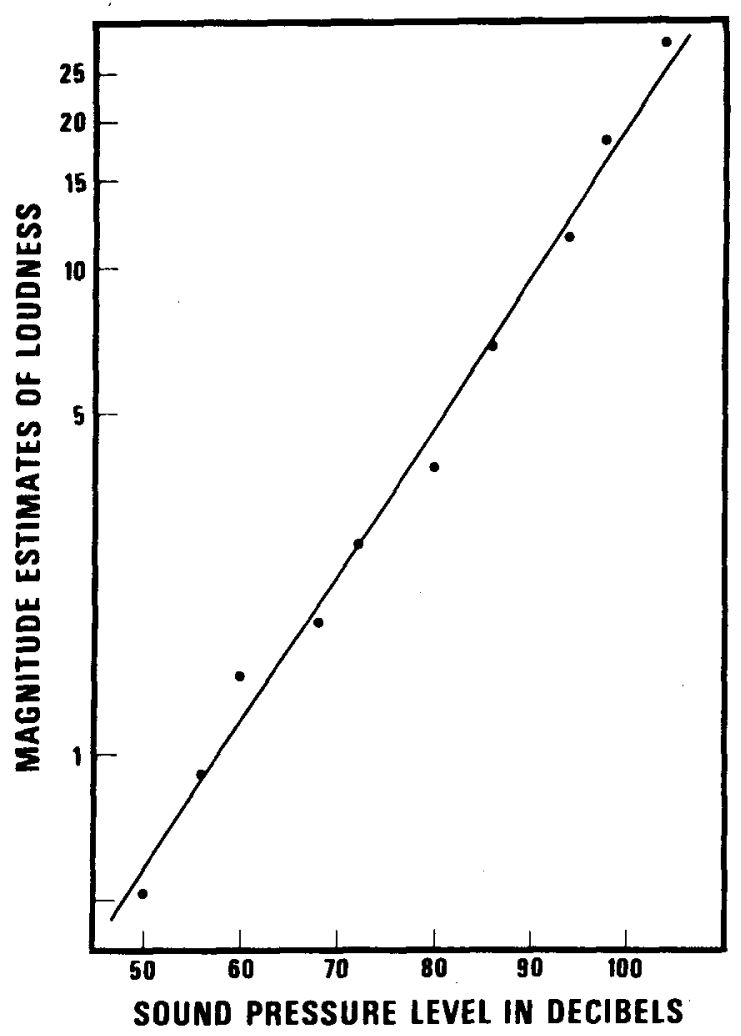

Figure 3. Magnitude estimates of the loudnesses of single tones as a function of sound pressure level in $\log -\log$ coordinates. 
single ranking of the 45 pairs would be consistent with each of the 540 binary comparisons. Therefore, a ranking was sought that would minimize the number of instances in which the binary comparisons were not in agreement with the specified rank order of pairs. Nine different rankings of the 45 tone pairs were found which minimized the number of instances in which the rankings disagreed with the pair comparison data. ${ }^{2}$

Since there were no criteria on which to select one of the rank orderings as "better" or more "representative," one-dimensional spatial solutions were obtained for each of the nine rank orders. Hence, each rank order was used as an input to the nonmetric scaling program. This program, as mentioned above, produces numerical assignments (projection values, $\mathrm{P}$, on a line segment) for each of the 10 tones such that the difference in numerical assignments between any two tones best predicts the rank order of the 45 pairs used as input for the program. Furthermore, these projection values are unique up to addition and multiplication by a constant, i.e., these values constitute interval scale measurement.

The stress values for these nine sets of projections ranged from $1.9 \%$ to $2.6 \%$. Stress values this low indicate "good" to "excellent" agreement between the predicted and obtained rank order of loudness ratio judgments. Since the index of metric determinancy was estimated at above 0.99 in all cases, the projection values can be considered unique up to addition and multiplication by a constant, i.e., they represent interval scale measurement.

Visual inspection of the nine sets of projection values indicated that they were virtually identical. This is what we would expect if the observed violations of transitivity which forced the construction of nine separate rankings resulted from random error in the judgment process. Correlation coefficients for all 36 comparisons of the nine sets of projections confirmed the visual impression. The 36 correlation coefficients, rounded off to four decimal places, ranged from 0.9994 to 1.0000 . Since all nine solutions were essentially identical, the average projection value for each tone was determined and is used in the following analyses.

In Experiment 1, we investigated two hypotheses. The first was that the rank order of loudness ratio judgments was a monotonic function of the ratio of the loudnesses of the two tones in a pair. The equivalent assumption in this experiment is that, if the ratio of the loudnesses of the two tones in pair $A$ is greater than the ratio of the loudnesses of the two tones in pair B, then the subject would judge that the tones in pair A had a greater loudness ratio than the tones in pair B. If this assumption were true, the projection values obtained from a nonmetric analysis of the rank order of pairs constructed from the binary zomparisons should be linearly related to decibels of

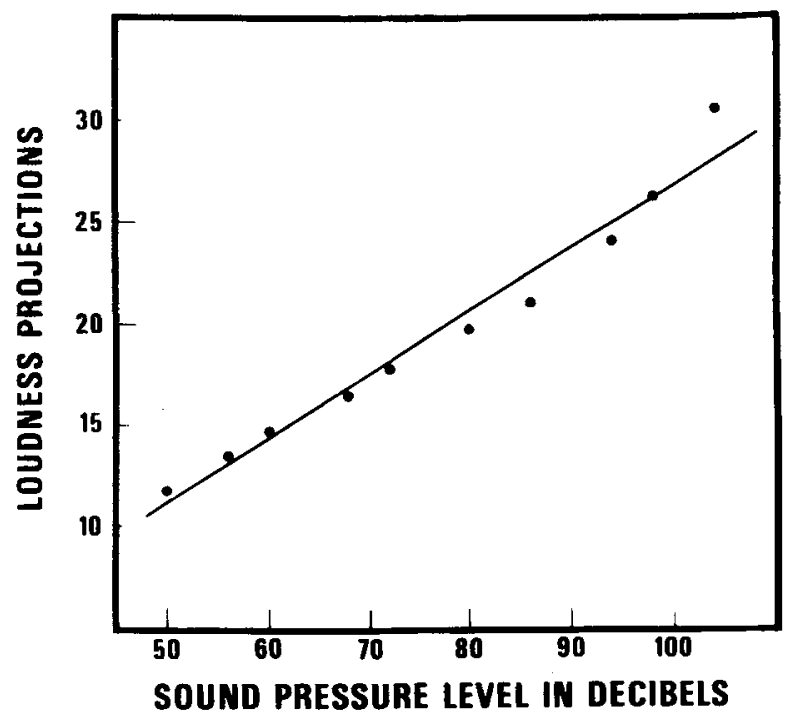

Figure 4. Loudness projections for 10 tones as a function of sound pressure level in decibels. The loudness projections were obtained from a nonmetric analysis of direct comparisons of loudness ratios for pairs of tones. A straight line in these coordinates would indicate that direct comparisons of loudness ratios were based on loudness ratios where loudness is a power function of intensity.

sound pressure. Figure 4 plots the projection values as a function of decibels of sound pressure. The straight line drawn through the points was fitted by the method of least squares. Note that the points in these coordinates are positively accelerated, although not to the same extent as the points in Figure 1. This positive acceleration (upward concavity) indicates that binary comparisons obtained from these subjects are inconsistent with either (1) the assumption that the comparisons are based on loudness ratios or (2) a power law representation for loudness.

If, on the other hand, Torgerson's conjecture is correct, binary comparisons should be based on loudness differences between the tones in a pair. Under this assumption, we would expect that the projection values, after suitable adjustment by a constant, would be linearly related to sound pressure in log-log coordinates (see above). Accordingly, an additive constant was found which maximized the squared Pearson correlation coefficient between $\log (P+b)$ and stimulus intensity in decibels. These projection values adjusted by the value of $b$ producing the maximum $\mathbf{r}^{2}$ are called the adjusted loudness projections. Figure 5 shows how the adjusted loudness projections vary as a function of sound pressure in $\log -\log$ coordinates. The best fitting straight line (method of least squares) provides a good fit to the data points $\left(r^{2}=0.993\right)$. Linearity in these coordinates suggests that the loudness ratio judgments were actually based on loudness differences rather than loudness ratios. The slope of the line (exponent of the power function) is approximately 0.14 . 


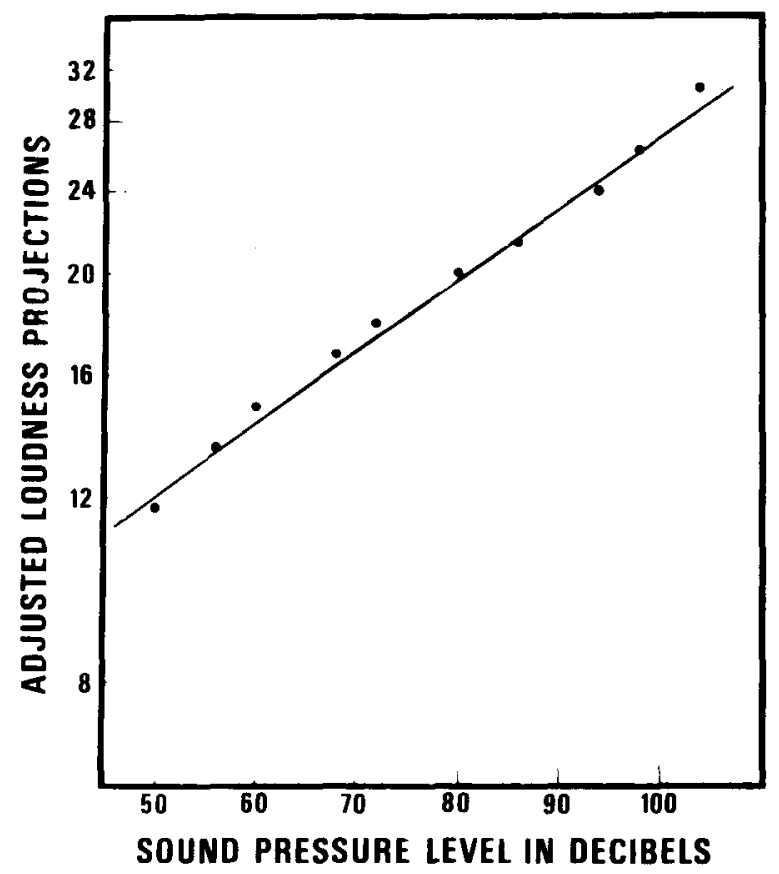

Figure 5. Adjusted loudness projections for 10 tones as a function of decibels of sound pressure. Notice that the ordinate is spaced logarithmically. The adjusted loudness projections were obtained from a nonmetric analysis which assumed that direct comparisons of loudness ratios were based on the loudness intervals for the two pairs of tones being compared. A straight line in these coordinates indicates that loudness ratios were based on loudness differences where loudness is a power function of intensity.

\section{DISCUSSION}

The present results indicate that, if loudness is a power function of intensity, both magnitude estimates of loudness ratios and direct comparisons of loudness ratios are based on loudness differences among the tones. This confirmation of Torgerson's conjecture follows from the results of the nonmetric analyses, which showed (1) that magnitude estimates of loudness ratios were monotonically related to the subtractive differences between the 0.23 power of the individual tones $\left[R_{i j}=m\left(I_{i}^{0.23}-I_{j}^{0.23}\right)\right]$, and (2) that binary comparisons of tone pairs based on judgments of loudness ratios were best predicted by determining which of the two tone pairs had the largest separation between the 0.14 powers of the tones in a pair; i.e., $R_{i j} \geqslant R_{k 1}$ iff $\mid\left(l_{i}^{0.14}\right.$ $\left.-\mathrm{Ij}_{\mathrm{j}}^{0.14}\right)|\geqslant|\left(\mathrm{I}_{\mathrm{k}}^{0.14}-\mathrm{I}_{1}^{0.14}\right) \mid$. The alternative model, that ratio judgments are based on a power transformation of the two corresponding sound pressure ratios, does not provide as good a fit to the data. This can be seen by making the following comparisons. If judgments of loudness ratios are based on loudness ratios, then, in Experiment 1, the predicted ranks, $\hat{R}_{\mathbf{i j}}=$ $m\left(L_{i} / L_{j}\right)=m\left(I_{i}^{n} / I_{j}^{n}\right)$, if loudness is a power function of intensity. Consequently, the pressure ratios of the tone pairs were used to predict the ratio judgments. The Spearman rank order correlation coefficient between the obtained and predicted ranks for the ratio model was 0.912 .

On the other hand, if ratio judgments are based on a difference model, then the predicted ranks, $\hat{\mathbf{K}}_{\mathrm{ij}}=$ $\mathrm{m}\left(\mathrm{I}_{\mathrm{i}}^{0.23}-\mathrm{I}_{\mathrm{j}}^{0.23}\right)$. The Spearman correlation coefficient between the predicted and obtained ranks for the difference model was 0.992 . It is clear from these correlation coefficients that judgments of loudness ratios are better predicted by the loudness difference model than by the loudness ratio model.

The relative adequacy of either the ratio model or the difference model can also be assessed for the pairwise comparisons in Experiment 2. For these data, the ratio model predicts that $\hat{\mathbf{R}}_{\mathbf{i j}} \geqslant \hat{\mathbf{R}}_{\mathrm{lm}}$ whenever $\mathrm{I}_{\mathbf{i}}^{\mathbf{n}} / \mathrm{L}_{j}^{\mathrm{n}} \geqslant$ $I_{\mathbf{i}}^{n} / I_{m}^{n}$. Of the 540 pair comparisons in this study, the ratio model predicted that 30 pairs should be judged as having identical loudness ratios. These pairs were not considered when assessing the fit of the data to the model. Of the 510 remaining comparisons, the ratio model failed in $13.5 \%$ of the cases. The difference model predicted that $\hat{\mathbf{R}}_{\mathrm{ij}} \geqslant \hat{\mathbf{R}}_{\mathrm{lm}}$ iff $\left|\left(\mathrm{I}_{\mathrm{i}}^{0.14}-\mathrm{I}_{\mathrm{j}}^{0.14}\right)\right| \geqslant$ $\left|\left(I_{1}^{0.14}-I_{m}^{0.14}\right)\right|$. Since this model predicted no ties in the pair comparisons, its adequacy was tested for all 540 pair comparisons. The loudness difference model failed to predict $9.8 \%$ of the pair comparison data. Note that in both cases (magnitude estimation of loudness ratios and binary comparisons of loudness ratios), the loudness difference model was better able to predict the subjects' judgments.

The one previous investigation of ratio judgments of loudness in which the data were complete enough to perform a nonmetric analysis is in substantial agreement with the present results. Richards (1974) had subjects estimate the ratios of monaural loudnesses in the 21 tone pairs constructible from seven $1,000-\mathrm{Hz}$ tones. Three different sets of seven tones were used spanning a range of 60,30 , or $15 \mathrm{~dB}$. Richards, in his analysis of the data, made the assumption that ratio estimates were based on psychological loudness ratios and that a power function described the relationship between loudness and intensity. This model, as we have seen in the introduction, implies that the log of the loudness ratio can be interpreted as distance along a line segment. Richards assumed that the $\log _{2}$ of the ratio judgments was the function relating distance in the spatial configuration to the loudness ratio judgments. In our analysis of his data, the less restrictive assumption, that the $\log$ of the ratio judgments was monotonic with distance in the spatial configuration, was made. Richards' analysis of the data showed that the ratio judgments were inconsistent with the ratio model and that, if a ratio model was to be employed, a two-dimensional representation of the data was necessitated. He (following McRobert, Bryan, \& Tempest, 1965) interpreted his data as suggesting that judgments of loudness ratios based on pairs either widely or marginally different in intensity were biased 
away from that predicted by a power law relationship with an exponent (as a function of sound pressure) of 0.60 .

Richards, however, did not explore whether a model based on loudness differences would better explain the data. We have reanalyzed his data assuming that loudness ratio judgments were a monotonic function of loudness differences and that a power law related loudness to intensity. Hence, we determined the additive constant (b) which maximized $r^{2}$ between $\log (P+b)$ and $d B$ of sound pressure where $P$ are the coordinate values from a nonmetric analysis of the ratio estimation data. As before, the $\left(P+b^{*}\right)$, where $b^{*}$ is the value of $b$ producing the maximum $r^{2}$ are called the adjusted loudness projections. Figure 6 (upper panels) show the relationship between $\log$ adjusted loudness projections and decibels of sound pressure for the three sets of tones. In each case, a straight line (method of least squares) provides a good fit to the data $\left(r^{2}=0.990,0.997,0.992\right.$, respectively $)$. The best estimates of the exponents of the corresponding power functions are $0.23,0.23$, and 0.44 , respectively, In contrast, the projection values (spaced linearly) are plotted as a function of decibels of sound pressure in Figure 6, lower panels. A straight line in these coordinates would indicate that the ratio model provided a good fit to the data. The points in the lower panels exhibit an upward concavity with the greatest degree of concavity associated with the largest range of tones. It should be noted that a straight line provides a better approximation to an arbitrary nonlinear function, the smaller the range of the nonlinear function. Therefore, we would expect, on a mathematical basis, that the goodness of fit of the linear approximation would increase as the stimulus range decreased, as is the case in Figure 6, lower panels.

An indication of the degree of fit of the two models to the data may be obtained by comparing each model's prediction of the rank order of ratio judgments, as we did for the data in Experiment 1. For the three sets of stimuli, the Spearman rank order correlations for the ratio model were $0.840,0.967$, and 0.971 , respectively. The equivalent rank order correlations for the difference model were 0.972 , 0.998. and 0.975. Again, in each case, the difference model provides a better description of the data. It should be noted that as stimulus spacing decreases, the extent of the differences in predicted rank order between the ratio and difference models decreases. The obtained differences in the Spearman correlation coefficients reflect this factor with the 2.5-dB spacing showing the smallest difference.

The present experiments and Richards' experiments suggest that loudness ratio judgments are based on loudness differences and that loudness is a power function of intensity. Five different estimates of

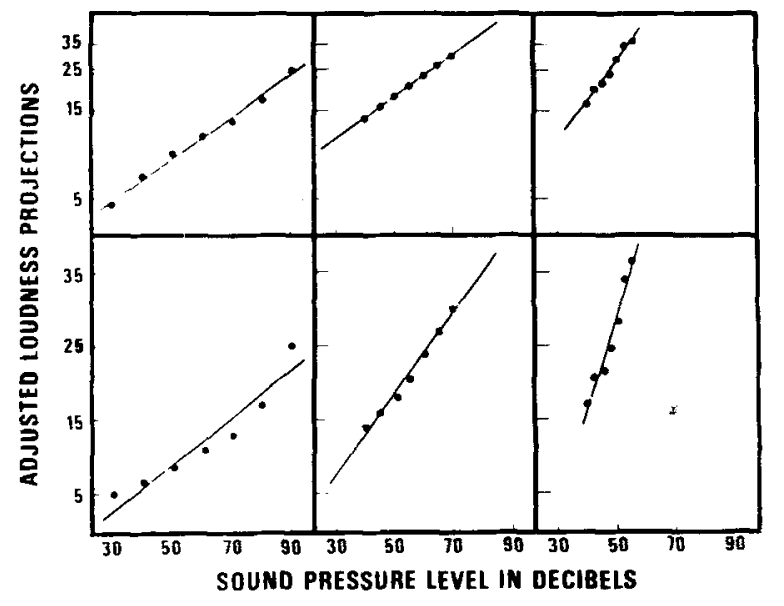

Figure 6. Adjusted loudness projections as a function of sound pressure level for $1,000-\mathrm{Hz}$ tones spaced $10 \mathrm{~dB}$ apart (left panels), $5 \mathrm{~dB}$ apart (middle panels), and $2.5 \mathrm{~dB}$ apart (right panels). Notice that the ordinate is spaced logarithmically in the upper panels and arithmetically in the lower panels. These adjusted loudness projections in each case were obtained from a nonmetric analysis of judgments of loudness ratios. A linear relationship in the upper panels indicates that judgments of loudness ratios are actually based on loudness differences (after Richards, 1974).

the exponent of the power function were obtained from the loudness ratio judgments. They are: Experiment 1, 0.23; Experiment 2, 0.14; Richards (1974), 0.23, 0.23, and 0.44 for ranges 60,30 , and $15 \mathrm{~dB}$. Three of these values are quite similar to the exponents of power functions obtained in nonmetric analyses of judgments based on loudness differences. These are: 0.27 (magnitude estimates of loudness differences for $1,200-\mathrm{Hz}$ tones; Parker \& Schneider, 1974; 0.24 (magnitude estimates of loudness similarity for 1,200-Hz tones; Parker \& Schneider, 1974); 0.29 (category estimates of loudness difference for $1,200-\mathrm{Hz}$ tones; Parker, Schneider, \& Valenti, Note 1); and 0.26 (paired comparisons of loudness differences for $1,200-\mathrm{Hz}$ tones; Schneider et al., 1974). If a common process is to explain the results of all of these experiments, it becomes necessary to explain the deviation of two of these exponents from the approximately 0.25 value which was obtained in six out of eight of the experiments reported above. ${ }^{3}$

The value of 0.44 for the exponent in Richards' third experiment may be due in part to the small separation of stimuli used in that study. Subjects in that experiment had difficulty discriminating tones separated by $2.5 \mathrm{~dB}$. To see that this was true. consider Richards' data on those stimulus pairs in which the two tones are identical. In the first two of Richards' studies (10 and $5 \mathrm{~dB}$ separation), 13 of 14 such pairs received mean ratio assignments of 1.00 while the 14th pair received a mean rating of 1.01 . In the third experiment ( $2.5 \mathrm{~dB}$ separation), none of the seven pairs of identicai tones received mean ratios of 1.00. This indicates that identical pairs were 
sometimes confused with pairs which contained tones differing by $2.5 \mathrm{~dB}$. There is, therefore, a suggestion that the subjects' numerical assignments for pairs with $2.5 \mathrm{~dB}$ separation are not trustworthy. Consequently, the 0.44 value for the exponent may have resulted from the error introduced by the subjects' lack of ability to discriminate, in this situation, tones separated by $2.5 \mathrm{~dB}$. A failure in discrimination of this sort could have artificially inflated the value of the exponent.

The low exponent, 0.14 , obtained from direct comparisons of loudness ratios (Experiment 2) may well have been caused by the difficulty the subjects had in making these judgments. In a debriefing session conducted after the experiment, all eight subjects reported difficulties in making these judgments, even though they had all had previous experience in magnitude estimation tasks. When specifically asked how they compared the tone pairs, all eight claimed that the judgment was very difficult, and seven of the eight subjects reported a common strategy they adopted for a subclass of the tone-pair comparisons. That strategy was to compare loudness "dissimilarities" and, if that provided no easy choice, to choose the tone pair containing the softer tones. Note that even when specifically instructed to base their judgments on loudness ratios, the subjects attended to loudness dissimilarities, a judgmental process which we might reasonably expect to be related to loudness differences. Second, for those tone pairs which were equally dissimilar, they chose the softer tones. The reason given by all seven subjects, for this strategy, was, in the words of one subject, "a constant difference on a smaller base gives you a larger ratio." That is, seven of the eight subjects, when asked to compare loudness ratios, used perceived differences and a knowledge of arithmetic to make their judgments. The effect of their choosing the softer of two comparable tone pairs as having the greater loudness ratio is to spread out the loudness values for the softer tones in the present analysis. Increasing the spread at the softer end of the scale results in a lowering of the exponent of the best fitting power function. Note that the effect is due solely to the subjects' knowledge of the way numbers behave and does not reflect the perceptual basis of the judgment. Presumably, then, without this bias, an exponent closer to 0.25 would have been obtained.

The eighth subject reported a strategy that suggested that his judgments were almost exclusively based on loudness differences even though he was attempting to judge loudness ratios. Naturally, his judgments deviated further from what would be predicted on the basis of the group representation (see Figure 5) than did the judgments of any of the other subjects. That is, the projection values, obtained from the nonmetric analysis were used to predict the 540 binary comparisons, i.e., the difference in projection values for the tones in pair $A$ was compared to the difference in projection values for the tones in pair $\mathbf{B}$, and the larger arithmetic difference was used to predict which loudness interval a subject should have judged as greater. Second, the actual binary judgments of the eight subjects were compared with the predicted binary judgments. The degree of agreement for each of the eight subjects was $74 \%$, $80 \%, 81 \%, 84 \%, 85 \%, 86 \%, 87 \%$. The lowest degree of agreement is for the subject with the aberrant strategy.

Another index of the difficulty or confusion that subjects experienced in trying to make direct comparisons of loudness ratios may be obtained from a consideration of the number of times two critical axioms concerning ratio judgments were violated. Krantz et al. (1971) have shown that both ratio judgments and difference judgments should satisfy the axioms of a positive difference structure. Two of these axioms are critical for our purposes in the sense that they may be tested by the data. The first states that the ratio judgments must be weakly ordered, i.e..

$$
(r, s) \geqslant(t, u) \text { and }(t, u) \geqslant(x, y) \text { implies }(r, s) \geqslant(x, y)
$$

The second, and more critical, axiom is the so-called monotonicity condition. This axiom states that if

$$
(a, b) \geqslant(x, y) \text { and }(b, c) \geqslant(y, z) \text { then }(a, c) \geqslant(x, z) \text {. }
$$

This axiom is illustrated in Figure 7. It says that pairs behave qualitatively like differences with respect to the ordering $\geqslant$. In the present experiment, there were 24 violations of transitivity as opposed to only 5 in the Schneider et al. (1974) experiment. Furthermore, the monotonicity axiom failed to hold for $5.7 \%$ of the cases in which it was applicable, whereas in the Schneider et al. (1974) experiment on binary comparisons of loudness difference judgments, it
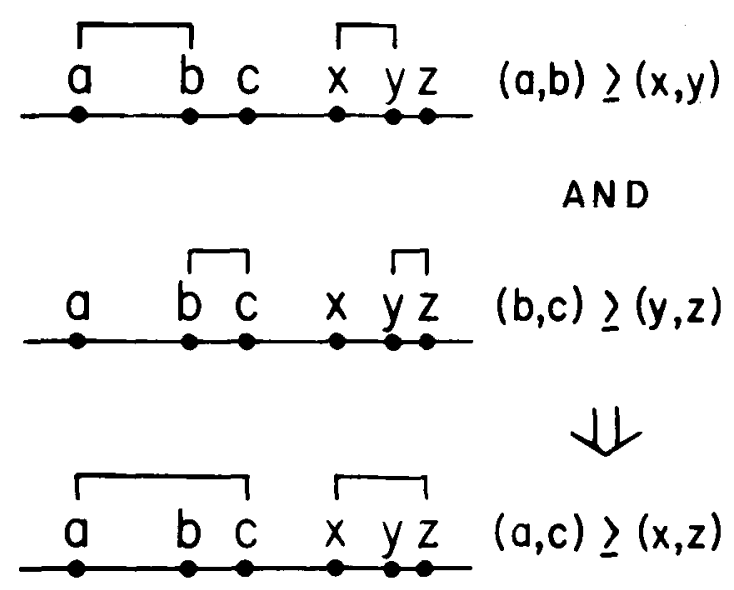

Figure 7. An illustration of the monotonicity axiom for points on a line. 
failed to hold in only $1.6 \%$ of the cases. The greater failure rate in the present experiment might be due to the rather complex strategies employed by subjects which involved direct comparisons of loudness intervals, and a partial correction for these judgments based on the subjects' knowledge of arithmetic.

It should be noted that there is at least one sensory continuum in which subjects can make consistent judgments of both sensory differences and sensory ratios. Parker, Schneider, and Kanow (1975) showed that judgments of line length differences were based on the differences between the 0.46 powers of physical lengths, while ratio estimates of line length pairs were based on a power transformation of the ratio of the two physical lengths in a pair. Hence, for at least one sensory continuum (line length or linear extent), two kinds of perceptual relationships are available to the subject (sensory differences and sensory ratios). However, when loudness is examined, it appears that Torgerson's (1961) conjecture, that there is one perceptual relationship but several monotonically related reporting strategies, is correct for monaural loudness. The results of the present and preceding experiments (Parker \& Schneider, 1974; Richards, 1974; Schneider et al., 1974; Parker, Schneider, \& Valenti. Note 1) indicate that only one perceptual relationship is defined for pairwise comparisons of tones, and that the effect of different instructions is simply to change, in a monotonic fashion, the reporting strategy employed by the subject but not the nature of the perceptual relationship reported. In addition, Birnbaum and Veit (1974), using a functional measurement analysis of ratio and difference judgments for pairs of lifted weights, concluded that, if a convergent scale criterion was employed, subjects do not distinguish differences from ratios. Hence, Torgerson's conjecture is supported for both loudness and lifted weights but not for line length.

The present results suggest a reconsideration of the taxonomy of perceptual continua proposed by S. S. Stevens (1957) in which these continua are divided into two classes: prothetic and metathetic. The present results and those of Parker et al. (1975) indicate a difference between two examples of prothetic continua (line length and loudness) when difference and ratio judgments of stimulus pairs are examined. The present division of sensory continua into prothetic and metathetic then needs to be reexamined.

The present results also raise some questions concerning the proper interpretation of the magnitude estimation experiment. If the subjects, when asked to judge or compare loudness ratios for pairs of tones, base their reports on loudness differences, how can subjects in a magnitude estimation experiment assign numbers to tones such that the ratios of the numbers reflect the ratios of the loudnesses? Furthermore, why do magnitude estimation experiments produce power functions, and why do these power functions have exponents approximately double those determined by nonmetric analyses? It appears that a reconsideration of the perceptual basis of the magnitude estimation experiment might be in order. Some data bearing on that reinterpretation will be taken up in a later paper.

\section{REFERENCE NOTE}

1. Parker, S., Schneider, B., \& Valenti, M. Nonmetric determination of the loudness function. Paper presented at the Eastern Psychological Association, 1973.

\section{REFERENCES}

Birnbaum, M. H., \& Veit, C. T. Scale convergence as a criterion for rescaling: Information integration with difference, ratio. and averaging tasks. Perception \& Psychophysics, 1974, 15, 7-15.

Carvellas, T., \& Schneider, B. Direct estimation of multidimensional tonal dissimilarity. Journal of the Acoustical Society of America, 1972, 51, 1839-1848.

EIsLER. H. Empirical test of a model relating magnitude and category scales. Scandinavian Journal of Psychology, 1962, 3 , 88-96.

Hellman, R., \& Zwislocki, J. Monaural loudness function at $1000 \mathrm{cps}$ and interaural summation. Joumal of the Acoustical Society of America, 1963, 35, 856-865.

Krantz. D. H., Luce, R. D., Suppes, P., \& Tversky, A. Foundations of measurement (Vol. I) Additive and polynomial representations. New York: Academic Press, 1971.

KruSKaL, J. B. Multidimensional scaling by optimizing goodness of fit to a nonmetric hypothesis. Psychometrika, 1964, 29, 1-27.

McRobert, H., Bryan, M. E., \& Tempest, W. Magnitude estimation of loudness. Journal of Sound Vibration, 1965, 2 , 391-401.

Marks, L. E. On scales of sensation: Prolegomena to any future psychophysics that will be able to come forth as science. Perception \& Psychophysics, 1974, 16, 358-376.

Parker. S., \& Schneider, B. Non-metric scaling of loudness and pitch using similarity and difference estimates. Perception \& Psychophysics, 1974, 15, 238-242.

Parker, S., Schneider. B., \& Kanow, G. Ratio scale measurement of the perceived lengths of lines. Journal of Experimental Psychology: Human Perception and Performance, 1975. 104, 195-204.

Richards, A. M. Non-metric scaling of loudness. I. 1000-Hz tones. Journal of the Acoustical Society of America, 1974, 56, 582-588. SCHNEIDER, B., \& LANE, H. Ratio scales, category scales, and variability in the production of loudness and softness. Journal of the Acoustical Society of America, 1963, 35, 1953-1961.

Schneider, B., Parker, S., \& Stein, D. The measurement of loudness using direct comparisons of sensory intervals. Journal of Mathematical Psychology, 1974, 11, 259-273.

SHEPARD, R. N. Metric structures in ordinal data. Journal of Mathematical Psychology, 1966, 3, 287-315.

SIEGEL. S. Non-parametric statistics for the behavioral sciences. New York: McGraw-Hill, 1956. Pp. 229-238.

Stevens, J. C.. \& Guirao, M. Individual loudness functions. Journal of the Acoustical Society of America, 1964. 36. 2210-2213.

StEvens, S. S. The relation of pitch to intensity. Journal of the Acoustical Society oj America, 1935, 6, 150-154. 
Stevens. S. S. The direct estimation of sensory magnitudeloudness. American Journal of Psychology, 1956, 69. 1-25.

Stevens, S. S. On the psychophysical law. Psychological Revien, 1957, 64, 153-181.

SteVens. S. S. On the new psychophysics. Scandinavian Journal of Psychology. 1960, 1. 27.35.

Stevens. S. S. Issues in psychophysical measurement. Psychological Review, 1971, 78, 426-450.

Stevens, S. S., \& Galanter, E. Ratio scales and category scales for a dozen perceptual continua. Journal of Experimental Psychology, 1957, 54, 377-411.

Stevens, S. S., \& Guirao, M. Loudness, reciprocality, and partition scales. Journal of the Acoustical Society of America, 1962. 34. 1466-1471.

Torgerson. W. S. Distances and ratios in psychophysical scaling. Acta Psychologica, 1961, 19, 201-205.

Young, F. W. Nonmetric multidimensional scaling: Recovery of metric information. Psychometrika, 1970, 35, 455-473.

\section{NOTES}

1. Marks (1974) has shown that category scale values. when suitably adjusted by an additive constant. are a power function of sound pressure. However. the exponent of the power function is much lower than the one obtained via magnitude estimation techniques. The nonlinearity of the two kinds of scales is due to this difference in exponents.

2. For details of the procedure used to determine the best rank orders. see Schneider et al. (1974).

3. Similar variation in the estimated value of the exponent also occurs for direct scaling techniques. Marks (1974) reviewed a number of ratio scaling experiments in which the estimated value of the loudness exponent ranged from 0.34 to 0.77 . Hence, the results from a single experiment may not be sufficient to establish the value of an exponent. Whether extreme deviations are due to random thuctuations or differences in procedure is often difficult to determine.

(Received for publication August 20, 1975; revision received January 5. 1976.) 